\title{
Editorial for March 2021 issue
}

\author{
S V Raghavan ${ }^{1}$
}

Published online: 24 March 2021

(C) CSI Publications 2021

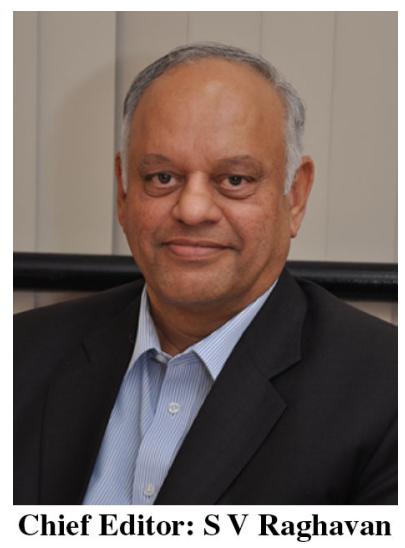

The March 2021 issue in your hands carries a series of interesting articles about education and institution. The institution in focus is the famous Sri Chandrasekharendra Saraswathi Viswa Maha Vidyalaya (SCSVMV in short), a Deemed University located in Enathur, Kanchipuram. The greatness of such institutions stay in the background most often in the din that surrounds us; mainly due to the postcolonial conditioning that has remained deep rooted in our education system. The New Education Policy 2020 brings in fresh air and encourages experiments. Around the same time, Covid-19 pushed the world to embrace ICT in full measure. In a recent conclave I participated to talk about the role of SCSVMV in the context of NEP 2020, the idea of focusing an issue highlighting the methods, practices, and successes, was born. One gets news about institutions that are in the urban areas that are funded by the

S V Raghavan

sv.raghavan@gov.in

1 Chennai, India
Government in good measure; but one often does not get to see India in its entirety. In this March 2021 issue, readers will get a comprehensive and coherent picture of experiences of a 25 year old institution founded for social upliftment of society in a rural area. Surprisingly, it fits well into our track on Education and Management of ICT. I hope the readers find the articles interesting and thought provoking. We are moving the journal in to era of open exploration of complex usage of the Information and Communication Technology; the reliability and resilience of the ICT based systems have become important in the changed scenario, i.e. post Covid. When intense usage comes from unexpected quarters such as Ayurveda, the ICT designer faces the problem of complexity of the system coupled with the simplicity expected by the user, which one can call Designers' Dilemma. Well, what we have is just the beginning and perhaps the tip of the iceberg. In my usual style, it is my pleasure to introduce briefly the ten articles of this issue.

1. Professor Karra Murthy in his article on 'Making of an Institution', explains the origin and growth of an institution that is rooted in Indian culture and tradition, while deftly combining the contemporary scientific thinking in the teaching learning process. Professor Murthy says, 'Every institution has a glorious beginning and SCSVMV is no exception. SCSVMV today is an university par excellence rooted in the Indian tradition, knowledge, and belief, but scientific in its thinking, as ordained by Adi Shankaracharya Ji Maharaj who is the fountainhead of knowledge and who has authored many voluminous works that explain from the working of a soul to the existence of the cosmos, in its entirety. We, at SCSVMV, are fortunate 
to be able to capture the spirit and essence of our institution in this paper'.

2. Professor Raghavan's musings on 'How to be an Effective Teacher', opens up one's mind to the vast tradition of India. Professor Raghavan says, 'Being a Teacher is a spiritual experience. One not only experiences happiness and satisfaction, but also imparts them to students who are listening. In this article, the author tries to capture the splendour of the classroom experience that practices the ideal form of teaching - learning process. The basis is the prescriptive methods given to this world by Adi Shankaracharya'.

3. Professors D K Subramanian et al., in their article, 'Behaviour Determines Technology', analyses threadbare the reductionism that has set in our education system and suggest a transformative change to holistic education. Professor D K Subramanian et al. has the following to say, 'We have spent five and four decades respectively, in the field of higher education. The information chronicled represents our collective understanding. We describe 'the desirable way' for generations to come, after witnessing changes for the better and for the worse. Our emphasis is on holistic view, be it learning, research, technology, commerce, or environment; when we say holistic, we mean in space and time. In our discourse, we are agnostic to belief (hypothesis) based dialectic logic and reasoning system and axiomatic (and hypothesis) based proving system that follows methodology assiduously'.

4. Professor S V Raghavan, Dr. Vasanth Kumar Mehta, and Professor $G$ Srinivasu in their article, Palm Leaf Manuscripts hold the knowledge of India dating back to several centuries. Therefore, Palm Leaf manuscripts are valued as Saaswathaiswaryam, implying eternal (Saaswath [1] ) wealth (Aiswaryam [2] ). But the Palm Leaf manuscripts are in different conditions- brittle, moth-eaten, edge-damaged, portions broken, etc. Besides, the leaves themselves are in bundles, that too out of order. The challenge for our generation is to design a methodology to capture the contents, recreate the missing portion, and figure out a way to right-order the manuscripts. Alongside, use modern technology for storage and retrieval, with emphasis on relevance to the requirement posed in the search query.

5. Transcript of the Keynote Speech given on the occasion of Unnat Bharath Abhiyan program by Professor S.V. Raghavan, Vice Chancellor, SCSVMV

6. Changes in the operating ambience is normally welcome. But the change world is facing is accompanied by an unacceptable reason called Covid virus spread. However, educational institutions, while observing the norms imposed by various administrations, have managed to do an excellent job of 'teaching' against the odds. Moreover, everyone was caught by surprise. Overnight the world changed. We decided to understand the reactions from our faculty to the challenge arising out of Covid, and also use of terminologies such as online, virtual, reality, perception, understanding, and so on. The result is this paper, which is a collection of 'reactions'.

7. As part of the programme to develop low-cost instruments for use in educational institutions, we have developed a heat-flux differential scanning calorimeter (HF-DSC). We have used low cost components for building the parts of the calorimeter and they were fabricated by the local industries. The paper describes the design and construction of the calorimeter and measurement electronics in detail. The calorimeter was calibrated for temperature and enthalpy measurements using high purity metal standards. The results indicate that the on-set temperatures of thermal events can be measured with an accuracy of $\pm 0.7 \mathrm{o} \mathrm{C}$ and the enthalpy values within $\pm 3 \%$. The sensitivity of the instrument is about 4 to 5 times lower than that obtained in a typical commercial DSC unit.

8. We have developed an educational model of power compensated differential scanning calorimeter (PCDSC) with a few modifications for the science students of academic institutions. In order to demonstrate the instrumentation and analytical aspects involved in the system, all the building blocks are constructed using local industry and are explained in detail. A few deviations from the conventional model have been attempted without compromising the basic calorimetric concept. This is done with a due consideration for cost factor and also to keep the openness in the development. The paper discusses the calibration procedure of the modified system to arrive at the on-set temperature and enthalpy of the samples. We are able to measure the onset temperature within $\pm 1.18{ }^{\circ} \mathrm{C}$ and the enthalpy values using this proto version with an error $5 \%$.

9. A novel Schiff base of N1,N5-Bis(salicylidene) tetraethylene pentamine (BSTEPA) was synthesized by using reflux method. The prepared molecule has been characterized using $1 \mathrm{H}$ NMR and IR spectral methods. The complete vibrational characterization of the molecule was performed using experimental (FTIR and FT-Raman) spectra and calculations at Density Functional Theory (DFT) level. In order to perform a detailed vibrational spectroscopic analysis of BSTEPA, Fourier Transform Infrared (FTIR) and Fourier Transform Raman (FT-Raman) spectra were recorded in condensed phase and used as such. Density Functional Theory calculations in the B3LYP/6-31G** 
level have been carried out on the title molecule in order to determine the optimized geometry and vibrational wavenumbers. The ultraviolet visible (UV-Vis) spectra of BSTEPA were recorded in the range of $300-500 \mathrm{~nm}$ for various solvents. The natural bond orbital and frontier molecular orbital analyses were also performed at same DFT level of calculations.

Alignment with National Education Policy 2020 of the Government of India, rooted in tradition and culture, discovering the vast potential within, and indigenous scientific instrument creativity span the March 2021 issue of CSI Transactions on ICT.

Happy Reading.

Professor S V Raghavan

Chief Editor

Publisher's Note Springer Nature remains neutral with regard to jurisdictional claims in published maps and institutional affiliations. 